
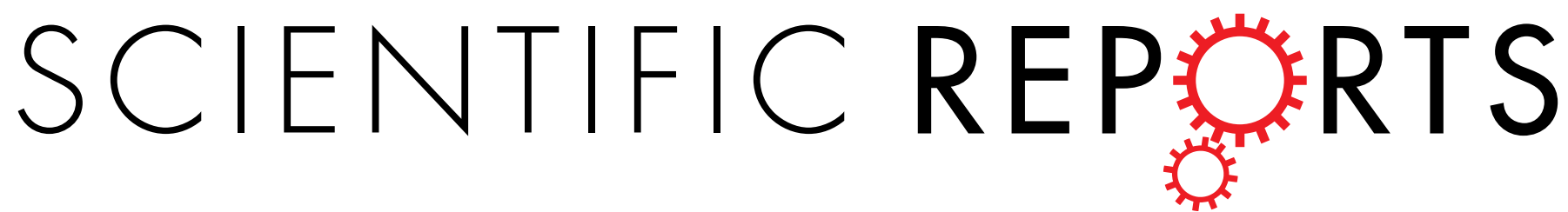

OPEN MicroRNA-214 Mediates

\title{
Isoproterenol-induced Proliferation and Collagen Synthesis in Cardiac Fibroblasts
}

\author{
Min Sun*, HaiyiYu*, Youyi Zhang, Zijian Li \& Wei Gao
}

The action of $\beta$-adrenergic receptors ( $\beta$-ARs) induces cardiac fibroblast (CF) proliferation and collagen synthesis and is a major source of the cardiac fibrosis caused by various diseases. Recently, microRNA-214 (miR-214) was found to play an important role in the pathogenesis of cardiac remodelling. In the present study, we examined the role and the underlying mechanism of miR-214 in isoproterenol (ISO, a $\beta$-AR agonist)-induced CF proliferation and collagen synthesis. The expression of miR-214 was increased in both ISO-mediated fibrotic heart tissue and fibroblasts. Downregulation of miR-214 by antagonists attenuated the proliferation and collagen synthesis in ISO-treated CFs. Using bioinformatics analysis and luciferase assays, mitofusin2 ( $\mathrm{Mfn} 2$ ), a critical regulator of cell proliferation and tissue fibrosis, was identified as a direct target gene of miR-214; this result was confirmed by western blot analysis. Additionally, corresponding to the upregulation of miR-214, the expression of Mfn2 was downregulated in the fibrotic heart and fibroblasts. Furthermore, the downregulation of miR-214 inhibited the activation of ERK1/2 MAPK signalling induced by ISO treatment. In conclusion, our study demonstrated that miR-214 mediates CF proliferation and collagen synthesis via inhibition of Mfn2 and activation of ERK1/2 MAPK signalling, which provides a new explanation for the mechanism of $\beta$-AR activation-induced cardiac fibrosis.

Cardiac fibrosis is an important pathological change occurring in cardiac remodelling following ischemic heart disease, hypertension, cardiomyopathy, and other diseases. This phenomenon contributes to the impairment of pump function and provides the basis for heart failure ${ }^{1}$. The proliferation of cardiac fibroblasts ( $\mathrm{CFs}$ ) and excessive deposition of extracellular matrix (ECM) proteins such as collagen types I and III are the major characteristics of cardiac fibrosis ${ }^{2} . \beta$-adrenergic receptors ( $\beta$-ARs), the dominant adrenergic receptors in the heart, have been reported to be excessively stimulated in various cardiovascular diseases ${ }^{3}$ and play a critical role in cardiac fibrosis ${ }^{4}$. Excessive $\beta$-AR stimulation can promote the proliferation and collagen synthesis of cardiac fibroblasts by activating ERK1/2 MAPK and p38 MAPK signalling, transactivating the epidermal growth factor receptor, and inducing the production of cytokines ${ }^{4-7}$. However, the function and mechanism of microRNAs in $\beta$-AR-mediated cardiac fibrosis remain unclear.

miRNAs are 18- to 25-nucleotide conserved noncoding RNAs that negatively regulate gene expression through mRNA cleavage or translational repression by base pairing with complementary sequences in the $3^{\prime}$ untranslated regions ( $3^{\prime}$ UTRs) of target genes. Recent studies have revealed that miRNAs play an important role in the pathogenesis of cardiac fibrosis 8 . Cardiac-specific deletion of the endonuclease Dicer, which is required for miRNA maturation, has been shown to result in cardiac hypertrophy and myocardial fibrosis9. Several miRNAs, including miR-133a $a^{10,11}$, miR-206 $6^{12}$, miR-2 $1^{13,14}$ and miR-29b ${ }^{15,16}$, have been reported to actively participate in cardiac fibrosis by controlling collagen synthesis and degradation, fibroblast proliferation, and the key signalling pathways regulating fibrosis. Moreover, a recent study demonstrated that $\beta$-ARs can regulate miRNA expression in the rat heart ${ }^{17}$, suggesting that miRNAs may mediate $\beta$-AR-induced cardiac fibrosis.

Department of Cardiology, Peking University Third Hospital and Key Laboratory of Cardiovascular Molecular Biology and Regulatory Peptides, Ministry of Health, Key Laboratory of Molecular Cardiovascular Sciences, Ministry of Education and Beijing Key Laboratory of Cardiovascular Receptors Research Beijing 100191, China. *These authors contributed equally to this work. Correspondence and requests for materials should be addressed to Z.J.L. (email: lizijian@bjmu.edu.cn)orW.G. (email:weigao@bjmu.edu.cn) 
miR-214, a sensitive marker of cardiac stress, was found to be upregulated in hearts overactivated by $\beta$-ARs, using a miRNA array test ${ }^{17}$. This upregulation can provoke cardiac hypertrophy and heart failure ${ }^{18}$. In addition, recent studies have also reported that downregulation of miR-214 can attenuate unilateral ureteral obstruction (UUO)-induced renal fibrosis ${ }^{19}$. These studies suggest that miR-214 may play an important role in cardiac fibrosis induced by excessive stimulation of $\beta$-ARs.

In the present study, we explored the role and mechanism of miR-214 in isoproterenol (ISO, a $\beta$-AR agonist)-induced cardiac fibrosis. Our results show that miR-214 mediates ISO-induced proliferation and collagen synthesis in CFs by directly targeting Mfn2 and activating the downstream extracellular signal-regulated kinasemitogen-activated protein kinase (ERK1/2 MAPK) signalling pathway.

\section{Results}

miR-214 is upregulated in ISO-induced cardiac fibrosis. Previous studies have demonstrated that the expression of miR-214 is upregulated in the ISO-treated rat heart ${ }^{17}$; thus, we first examined whether the level of miR-214 also changes in an ISO-induced cardiac fibrosis model. In vivo, SD rats were treated with subcutaneous injection of ISO $(0.25 \mathrm{mg} / \mathrm{kg})$ once a day for 7 consecutive days, and histological staining with Sirius red and hydroxyproline quantification were then performed to evaluate cardiac fibrosis. Compared with the control group, the cardiac interstitial fibrosis area and collagen content increased following ISO injection (Fig. 1A,B), demonstrating that the cardiac fibrosis model was successfully established. Furthermore, the level of miR-214 was significantly increased in the fibrotic heart (Fig. 1C). In vitro, miR-214 was upregulated by ISO in a dose- and time-dependent manner when compared to the control (Fig. 1C,D), suggesting that miR-214 may play a role in cardiac fibrosis.

Downregulation of miR-214 attenuated ISO-induced proliferation and collagen synthesis of cardiac fibroblasts. To explore the role of miR-214 in cell proliferation and collagen synthesis, CFs were transfected with agomir-214 and antagomir-214 to overexpress and knock down miR-214, respectively. At $48 \mathrm{~h}$ post-transfection, miR-214 levels were measured by quantitative RT-PCR. Transfection with $50 \mathrm{nM}$ agomir-214 augmented miR-214 expression, whereas transfection with $100 \mathrm{nM}$ antagomir-214 reduced miR-214 levels significantly (Fig. 2A,B).

The BrdU incorporation assay was applied to detect cell proliferation, and the ${ }^{3} \mathrm{H}$-Proline incorporation assay and collagen I and collagen III mRNA quantification assays were used to evaluate the collagen synthesis ability of CFs. Compared with negative control group, knockdown of miR-214 significantly decreased cell proliferation, collagen synthesis rate, collagen I and collagen III mRNA expression of cultured CFs (Fig. 3A-D), while overexpression of miR-214 increased cell proliferation and collagen synthesis (Fig. 3E,F).

As shown in Fig. 4, ISO treatment increased cell proliferation, ${ }^{3} \mathrm{H}$-Proline incorporation and the mRNA levels of collagen I and collagen III in cultured CFs. In contrast, downregulation of miR-214 attenuated the increase in ISO-induced cell proliferation and collagen synthesis, demonstrating that miR-214 mediates ISO-induced cardiac fibroblast proliferation and collagen synthesis (Fig. 4A-D). However, miR-214 overexpression did not further accelerate the increase in cell proliferation and collagen synthesis when compared to ISO treatment (Fig. 4E-H).

Mfn2 is the molecular target of miR-214. Because miRNAs negatively regulate gene expression at the post-transcriptional level by directly binding to the complementary sequences in the 3'UTRs of target genes, we searched several bioinformatics databases, including TargetScan (http://www. targetscan.org/), miRanda (http:// www.microrna.org/microrna/home.do), PITA (http://genie.weizmann.ac.il/index.html) and miRWalk (http:// www.umm. uniheidelberg.de/apps/zmf/mirwalk/), to identify the putative miR-214 target genes involved in the regulation of proliferation and collagen synthesis in CFs. The analysis predicted the sequence position 952-958 in the $3^{\prime}$ UTRs of mitofusin2 (Mfn2) as a putative miR-214 binding site (Fig. 5A). This sequence is broadly conserved in human, mouse and rat models.

To identify whether Mfn2 is a direct target of miR-214 through this specific binding site, we constructed a wild-type luciferase reporter gene vector containing the $3^{\prime} \mathrm{UTR}$ of Mfn2 and a mutant vector with several mutations in the miR-214 binding site; we then co-transfected the reporter with agomir-214 in $293 \mathrm{~A}$ cells. Compared with the control, the luciferase activity of the wild-type group was significantly lower, whereas the mutant group was not affected when miR-214 was overexpressed Fig. $5 \mathrm{~B}$.

In addition, real-time PCR and western blotting analysis were applied to investigate the effects of miR-214 on both Mfn2 mRNA and protein expression. The outcomes showed that the mRNA level of Mfn2 was not affected, but the protein level was correspondingly decreased or increased when miR-214 was overexpressed or downregulated (Fig. 5C,D), indicating that miR-214 negatively regulates Mfn2 expression by translational inhibition. Moreover, in accordance with the upregulation of miR-214, the endogenous Mfn2 protein level was decreased in both the ISO-induced cardiac fibrotic heart (in vivo) and in the CFs (in vitro), suggesting that Mfn2 levels are reduced by miR-214 during the pathogenesis of cardiac fibrosis.

Inhibition of miR-214 reduces ISO-induced activation of ERK1/2-MAP kinase signalling. Previous studies have demonstrated that Mfn2 prevents vascular smooth muscle cell proliferation by inhibiting the Ras-RafERK1/2 signalling pathway via direct binding with both Ras and Raf ${ }^{20}$. ERK-MAP kinase signalling is a critical pathway that regulates CF proliferation and collagen synthesis ${ }^{4,5}$. Thus, we investigated the effect of miR-214 on ERK1/2 phosphorylation levels induced by ISO treatment.

As expected, downregulation of miR-214 significantly inhibited the increase of ERK1/2 phosphorylation induced by ISO treatment (Fig. 6A). In contrast, overexpression of miR-214 further promoted the increase in ERK1/2 phosphorylation (Fig. 6B). 
A

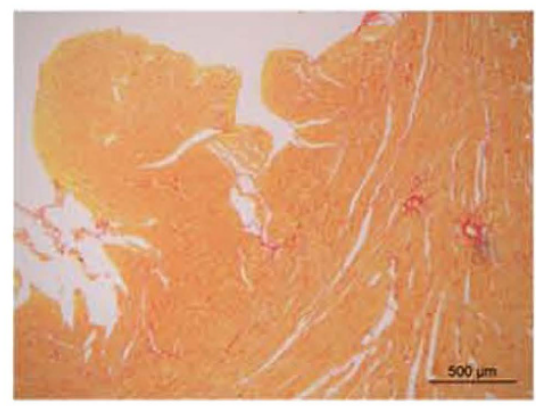

C

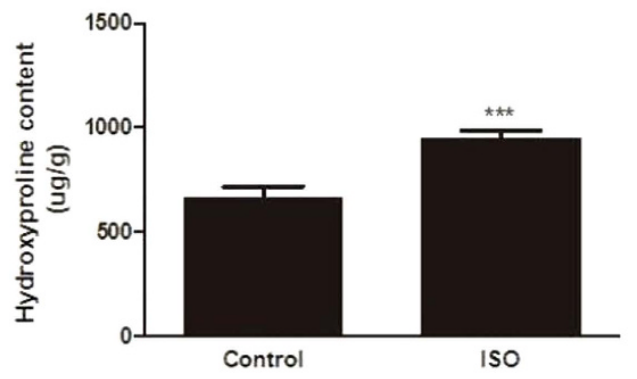

$\mathbf{E}$

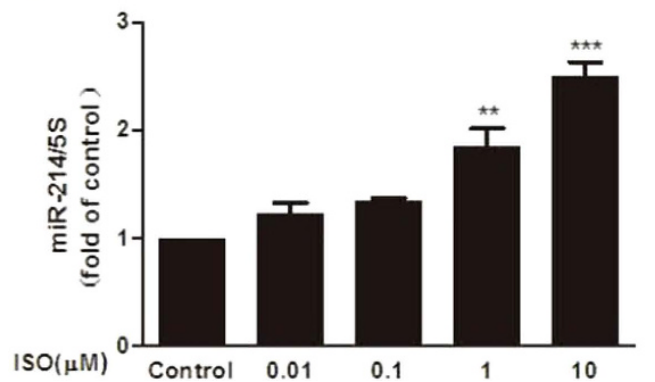

B

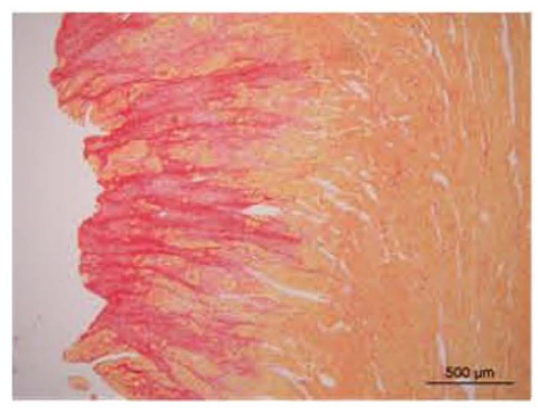

D

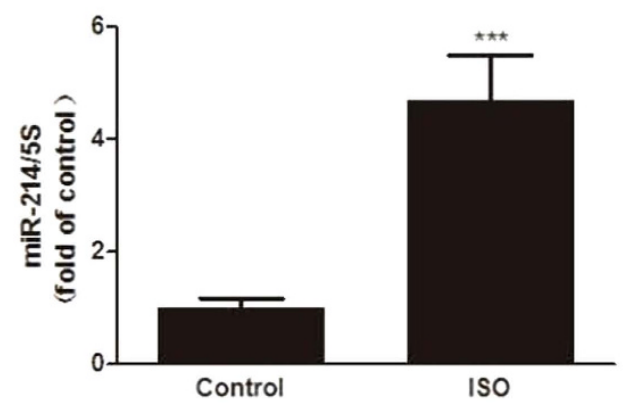

$\mathbf{F}$

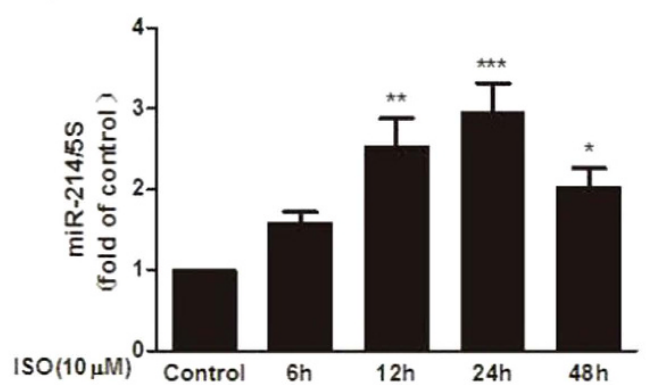

Figure 1. miR-214 is upregulated in ISO-induced fibrotic heart tissue and CFs. (A) Histopathological features of collagen deposition by Sirius red staining of heart sections from ISO-treated rats (100X magnification). (B) ISO enhanced the hydroxyproline content in cardiac tissue ( $\mathrm{n}=7$ for each group, mean \pm SEM, ${ }^{* * *} \mathrm{P}<0.001$ vs. Control). (C) The expression of miR-214 was increased in the myocardium of rats treated with ISO for 7 days compared to the control $\left(n=7\right.$ for each group, mean $\pm S E M$, ${ }^{x * *} \mathrm{P}<0.001$ vs. Control). (D) CFs were incubated with various concentrations of ISO for $24 \mathrm{~h}$, and miR-214 levels were measured by quantitative real-time PCR. (E) CFs were treated with $10 \mu \mathrm{M}$ ISO at the indicated time points $\left(\mathrm{n}=5\right.$, mean \pm SEM, ${ }^{*} \mathrm{P}<0.05,{ }^{* *} \mathrm{P}<0.001,{ }^{* * *} \mathrm{P}<0.001$ vs. Control).

\section{Discussion}

MiRNAs widely participate in the pathogenesis of cardiac fibrosis ${ }^{8}$. In the present study, we found that miR-214 was significantly upregulated in a cardiac fibrosis model induced by ISO, a $\beta$-AR agonist. Downregulation of miR-214 attenuated ISO-induced cardiac fibroblast proliferation and collagen synthesis, indicating that the $\beta$-AR-induced cardiac fibrosis was partly mediated by a pathway that involved miR-214, which may provide new insight to elucidate the mechanism of $\beta$-AR-induced cardiac fibrosis.

Previous studies have revealed that miR-214 expression is significantly increased in the heart tissues of patients with ischemic heart disease, dilated cardiomyopathy and atherosclerosis ${ }^{21}$ as well as in the serum of chronic heart failure patient $\mathrm{s}^{22}$. Similar results have also been obtained in animal models, where miR-214 is upregulated in both hypertrophic and failing heart tissues and cardiomyocytes ${ }^{22-25}$. Here, we found that miR-214 was significantly upregulated in $\beta$-AR-induced cardiac fibrotic heart tissues and fibroblasts. These studies indicate that miR-214 may serve as a biomarker or potential therapeutic target for cardiac remodelling.

In the cancer research field, miR-214 has been considered as a critical regulator of cell survival and proliferation. However, the conclusions thus far are controversial and depend on the tissues and target genes. In ovarian cancer and pancreatic cancer ${ }^{26,27}$, miR-214 has been found to act as an oncogene to promote cell proliferation and cisplatin 
A

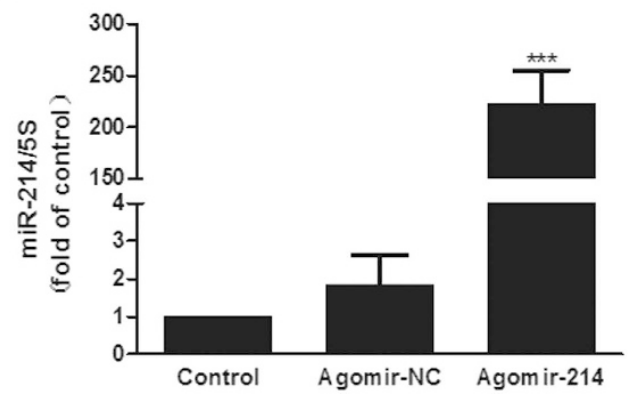

B

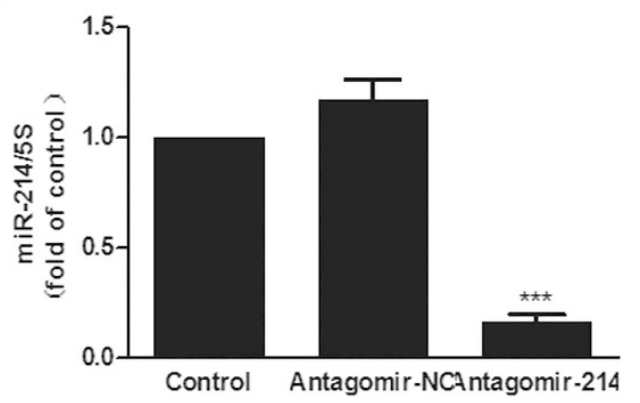

Figure 2. Overexpression or knockdown of miR-214 in CFs. (A) Transfection of agomir-214 (50 nM) in CFs significantly increased the expression of miR-214, as determined by quantitative real-time PCR. (B) Transfection of antagomir-214 $(100 \mathrm{nM})$ in CFs significantly decreased the expression of miR-214 $(\mathrm{n}=3$, mean $\pm \mathrm{SEM},{ }^{*} \mathrm{P}<0.05,{ }^{* *} \mathrm{P}<0.001,{ }^{* * *} \mathrm{P}<0.001$ vs. Control). NC: negative control.

resistance via the downregulation of PTEN and activation of the PI3K/AKT signalling pathway. In contrast, miR214 serves as a tumour suppressor in cervical cancer, hepatocellular carcinoma and myeloma cells by inhibiting cell proliferation, invasion and migration ${ }^{28-30}$. In the present study, we demonstrated that miR-214 plays a positive role in cardiac fibroblast proliferation, indicating a tissue-selective effect of miR-214 on cell proliferation.

In vivo studies, miR-214 has been reported to participate in the process of negative cardiac remodelling because overexpression of miR-214 induces cardiac hypertrophy and cardiac dysfunction whereas inhibition of miR-214 can prevent cardiac hypertrophy, interstitial fibrosis, impairment of angiogenesis and cardiac dysfunction of heart failure $^{22,31}$. In the present study, cardiac fibroblasts were transfected with agomir-214 and antagomir-214 referred to the preliminary experiments and other literatures ${ }^{32-37}$ and we found that the downregulation of miR-214 attenuated ISO-induced collagen synthesis in rat cardiac fibroblasts, which provides more evidence for the negative role of miR-214 in cardiac remodeling and helps to elucidate the possible mechanism. However, the miR-214 genetic deletion aggravated ischemia reperfusion injury-mediated cardiac fibrosis ${ }^{24}$. These contradictory results may be due to the different stimuli and compensatory mechanisms activated in the persistent absence of miR-214. Given that there are thousands of miRNAs working together in complicated networks in vivo, the genetic deletion of miR-214 may lead to compensation during development and thus may not reflect the real function. Moreover, the stimulus type may also affect the outcome.

$\mathrm{Mfn} 2$ is a protein that localizes to the outer membrane of the mitochondria; this protein plays an essential role in maintaining the morphology and function of the mitochondria ${ }^{38}$ and is a crucial regulator in controlling cell proliferation and tissue fibrosis ${ }^{20,39}$. Both in vitro and in vivo studies have demonstrated an inhibitory role of Mfn2 in cell proliferation for vascular smooth muscle cells (VSMCs ${ }^{20}$. Furthermore, overexpression of Mfn 2 can alleviate ECM deposition in the diabetic rat kidney ${ }^{39}$. In our study, Mfn2 was found to decrease in fibrotic rat heart tissues and fibroblasts and was negatively regulated by miR-214. This result indicates that miR-214-mediated cell proliferation and collagen synthesis occur at least partly via downregulation of Mfn2.

ERK1/2 MAPK signalling is a well-known modulator of CF growth and activation in the cardiovascular system. It has been demonstrated that $\beta$-AR agonists, such as ISO, can promote cardiac fibroblast proliferation and collagen synthesis by ERK1/2 MAPK activation ${ }^{4,40,41}$. Moreover, Mfn2 has been demonstrated to inhibit the Ras-Raf-ERK1/2 signalling pathway by directly binding with both Ras and Raf ${ }^{20}$. Therefore, we supposed that ERK1/2 MAPK signalling might be involved in miR-214-mediated CF activation. In line with our hypothesis, transfection of synthetic miR-214 antagonist led to a significant decrease in ISO-induced ERK1/2 MAPK activation, indicating that miR-214 could be a critical regulator of ERK1/2 MAPK activity in CFs.

In summary, our study reveals that ISO treatment increases the expression of miR-214 in both cultured neonatal fibroblasts and rat heart tissue. The upregulation of miR-214 can mediate ISO-induced proliferation and collagen synthesis in cardiac fibroblasts by regulating the target gene Mfn 2 and its downstream ERK1/2 signalling pathway. Our research demonstrates that miR-214 is a new regulator of $\beta$-AR-mediated cardiac fibrosis and may serve as a novel therapeutic target in cardiac fibrosis.

\section{Methods}

Cardiac fibrosis model. The animal care and experimental procedures involved in this study were approved by the Institutional Animal Care and Use Committee of Peking University Health Science Center (LA2010-037) and adhered to the American Physiological Society's "Guiding Principles in the Care and Use of Vertebrate Animals in Research and Training".

A cardiac fibrosis model was induced in 180- to 200-g male Sprague-Dawley rats by subcutaneous injection of ISO $(0.25 \mathrm{mg} / \mathrm{kg})$ once a day for 7 consecutive days. A corresponding control group of animals received an equivalent volume of physiological saline. The rats were sacrificed by cervical dislocation after deep anaesthesia with $2 \%$ isoflurane (Baxter Healthcare Corporation, New Providence, NJ, USA) at the end of day 7 . The walls of the ventricles were fixed with $4 \%(\mathrm{w} / \mathrm{v})$ paraformaldehyde, dehydrated, and embedded in paraffin. Ventricle sections were stained with Sirius red to observe interstitial fibrosis. The collagen content of the myocardial tissue was determined by a hydroxyproline assay kit (Cat. No. A030-1; Jiancheng, Nanjing, China). The remaining samples were frozen in liquid nitrogen for later use. 
A

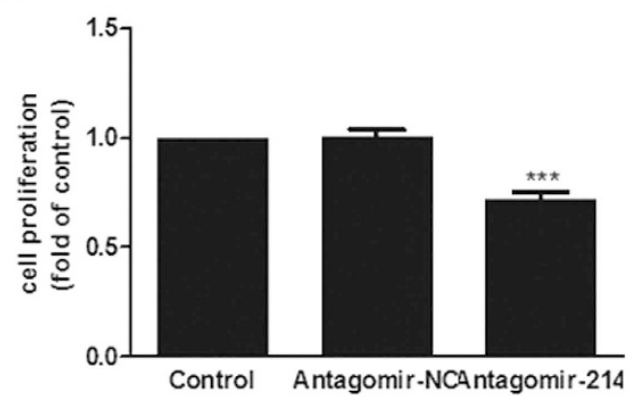

C

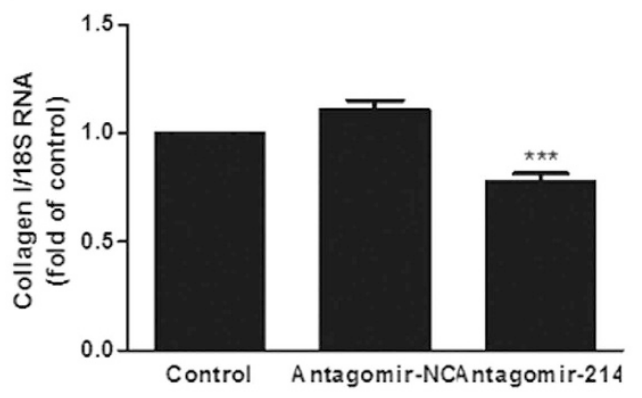

E

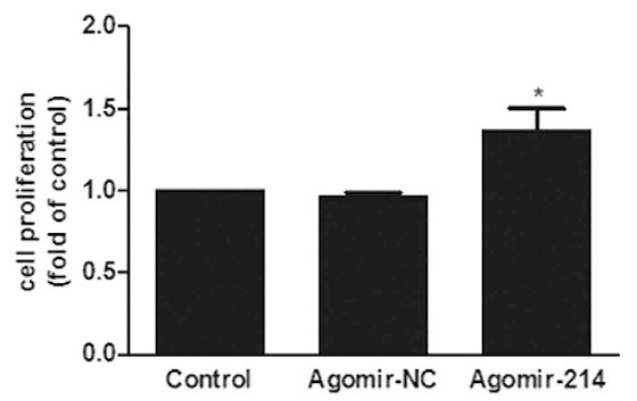

G

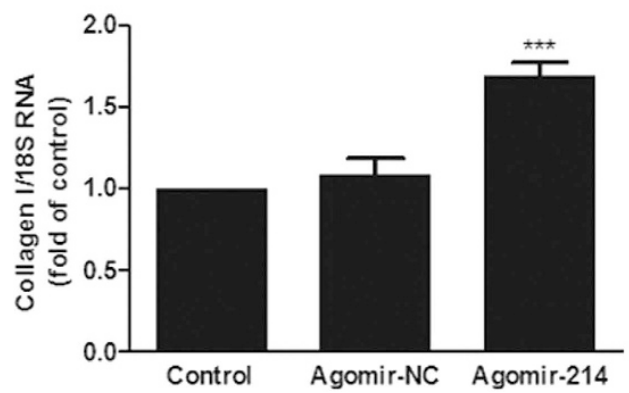

B

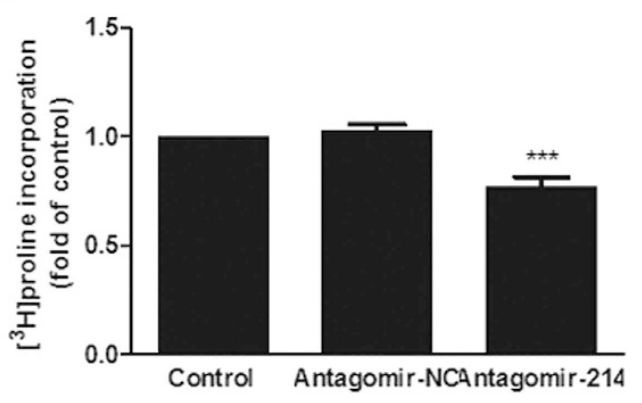

D

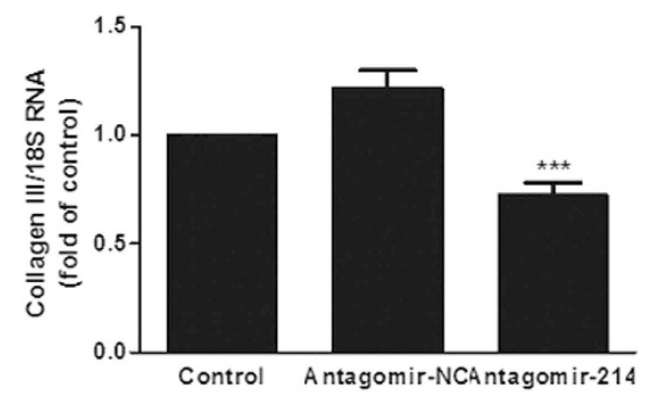

$\mathbf{F}$

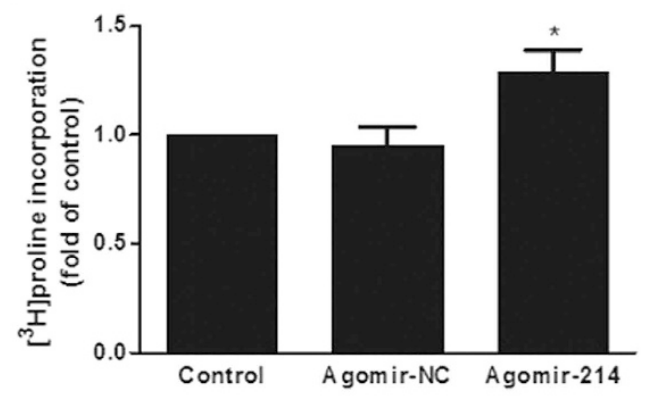

$\mathrm{H}$

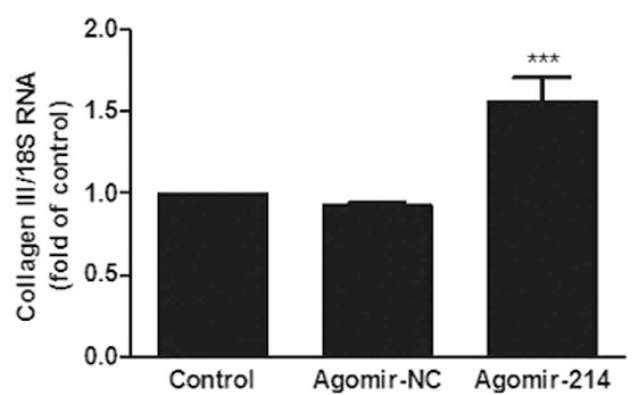

Figure 3. Downregulation of miR-214 attenuated the proliferation and collagen synthesis of CFs. Knockdown of miR-214 significantly decreased the proliferation (A), collagen synthesis rate (B) and the expression of collagens I and III (C,D) in CFs. Overexpression of miR-214 increased the proliferation (E), collagen synthesis rate $(\mathbf{F})$ and the expression of collagens I and III $(\mathbf{G}, \mathbf{H})$ in CFs. $(\mathrm{n}=3-4$, mean \pm SEM, ${ }^{\star} \mathrm{P}<0.05,{ }^{* * *} \mathrm{P}<0.001$ vs. NC).

Cell culture. Neonatal cardiac fibroblasts were isolated from the hearts of 1- to 3-day-old Sprague-Dawley rats. Briefly, a central thoracotomy was performed after the neonatal rats were deeply anaesthetized with $1.0 \%$ isoflurane. The hearts were quickly excised and immediately embedded in freezing Hanks' solution. Cardiac fibroblasts were isolated from the rat hearts by enzymatic digestion with pancreatin and collagenase type II as described previously ${ }^{42}$, grown to $80 \%$ confluence and serum-starved for $24 \mathrm{~h}$ in serum-free medium before treatment with ISO $(10 \mu \mathrm{mol} / \mathrm{L})$ or transfection with agomir $(50 \mathrm{nmol} / \mathrm{L}$; RibioBio, Guangzhou, China) or antagomir $(100 \mathrm{nmol} / \mathrm{L}$; RibioBio) referred to the preliminary experiments and other literatures ${ }^{32-37}$. 
A

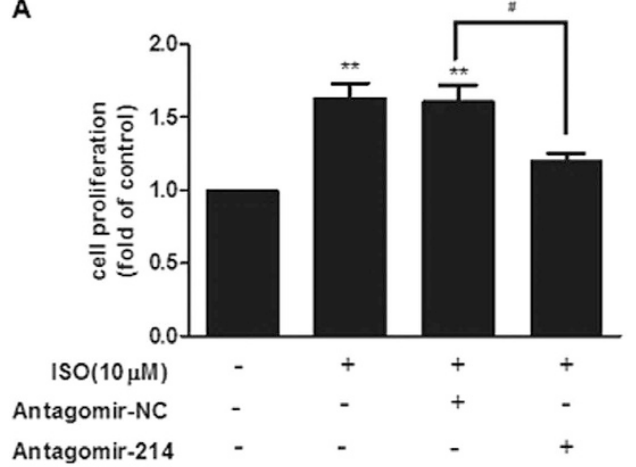

C

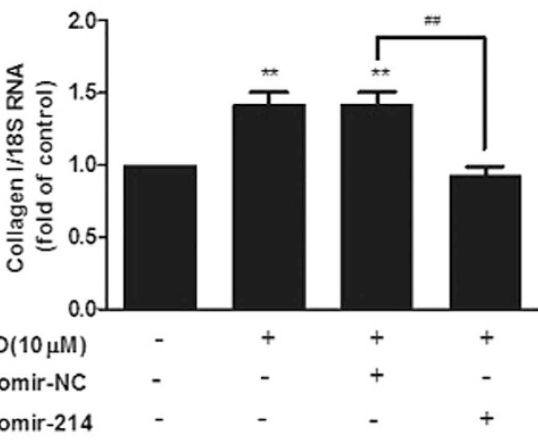

E

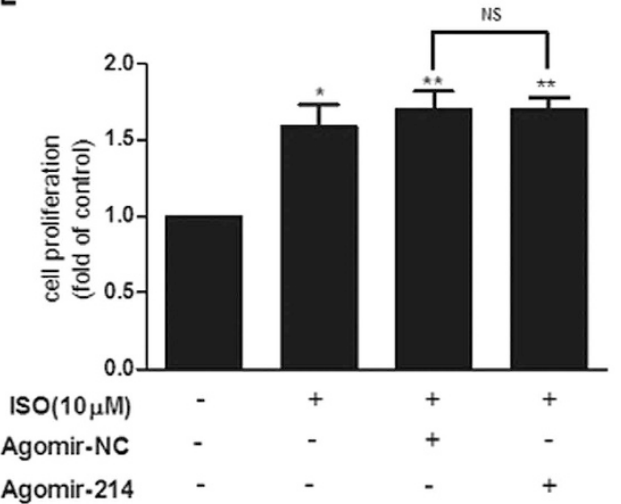

G

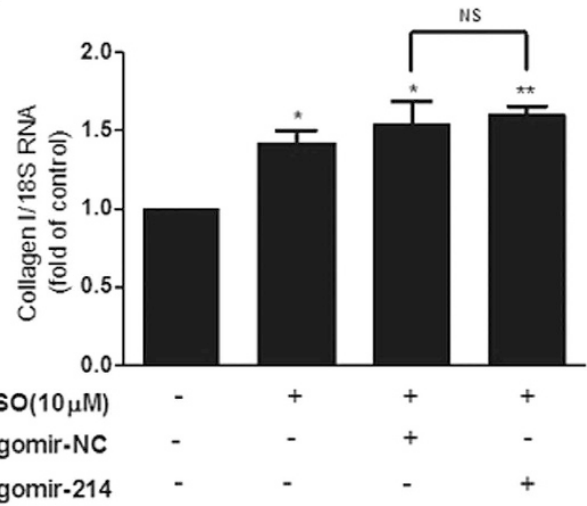

B

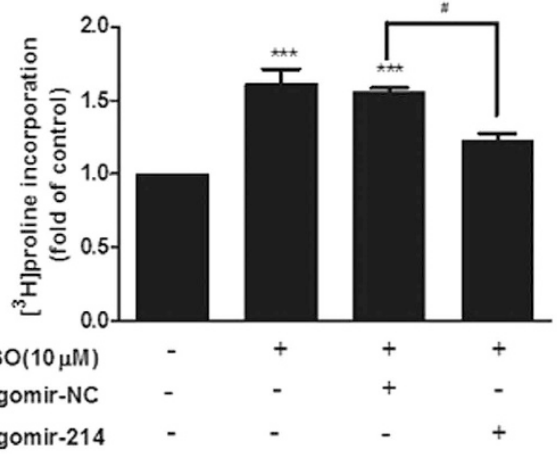

D

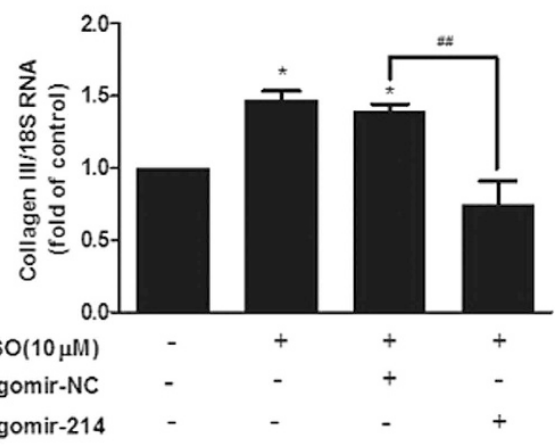

$\mathbf{F}$

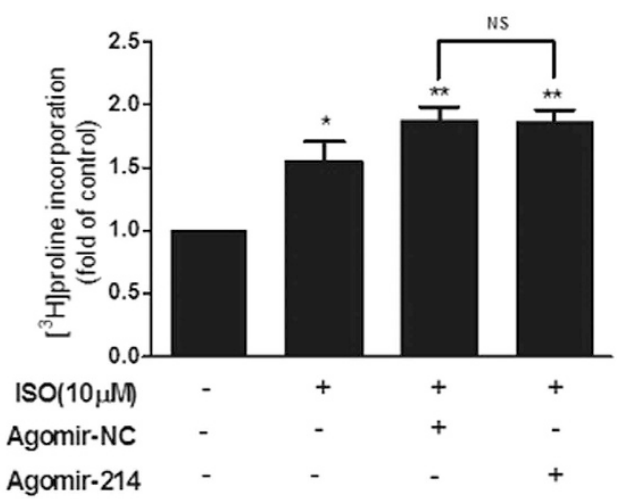

H

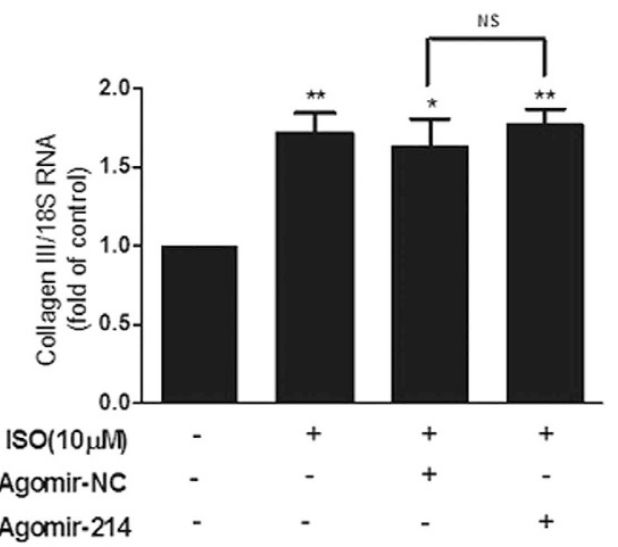

Figure 4. Downregulation of miR-214 attenuated ISO-induced proliferation and collagen synthesis of CFs. Knockdown of miR-214 significantly decreased ISO-induced proliferation (A), collagen synthesis rate (B) and the expression of collagens I and III (C,D) in CFs. Overexpression of miR-214 did not affect ISO-induced proliferation $(\mathbf{E})$, collagen synthesis rate $(\mathbf{F})$ or the expression of collagens I and III $(\mathbf{G}, \mathbf{H})$ in CFs. $(\mathrm{n}=3-4$, mean \pm SEM, ${ }^{*} \mathrm{P}<0.05,{ }^{* *} \mathrm{P}<0.001,{ }^{* * *} \mathrm{P}<0.001$ vs. Control, ${ }^{\#} \mathrm{P}<0.05$ vs. ISO $+\mathrm{NC},{ }^{\# \#} \mathrm{P}<0.01$ vs. ISO $\left.+\mathrm{NC}\right)$. 
A

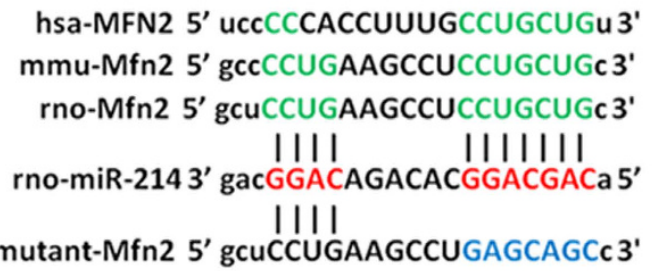

C
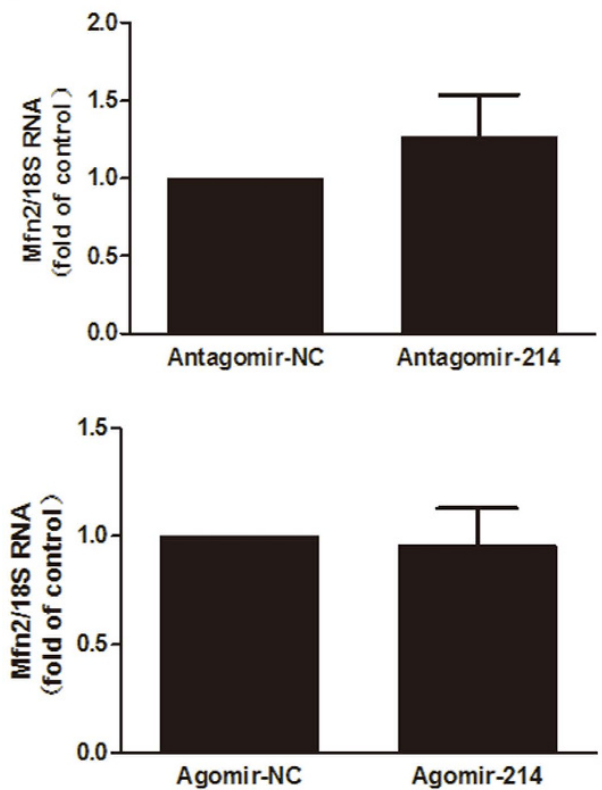

E
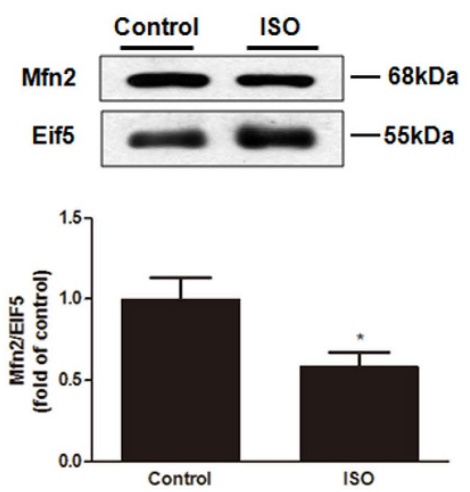

B

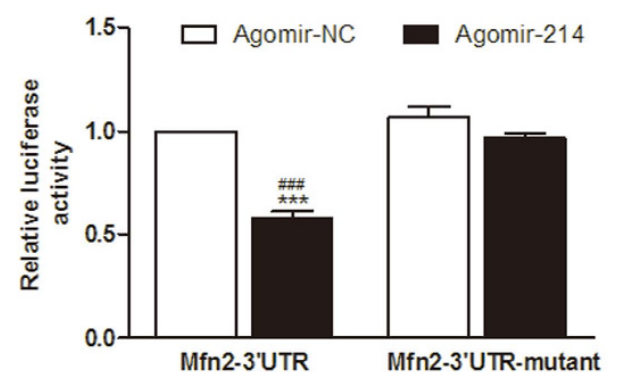

D
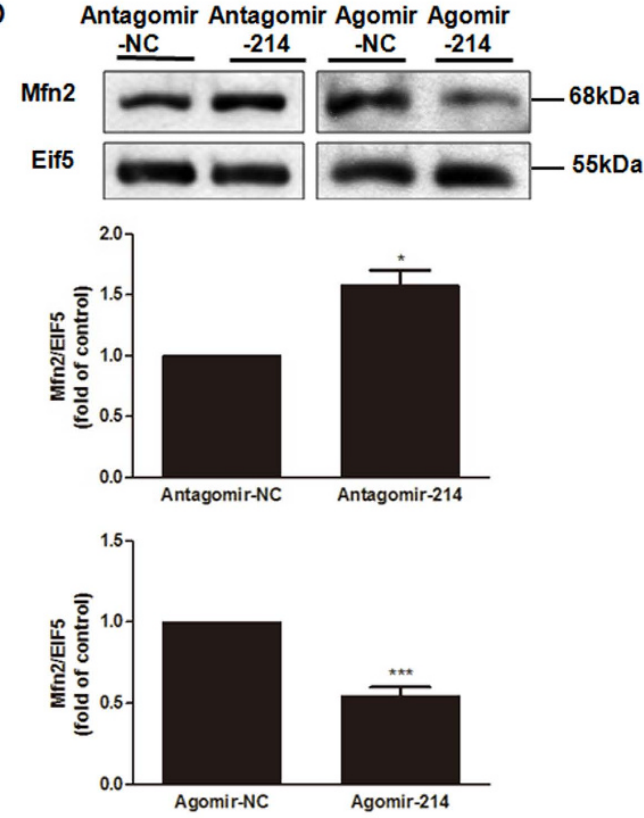

$\mathbf{F}$
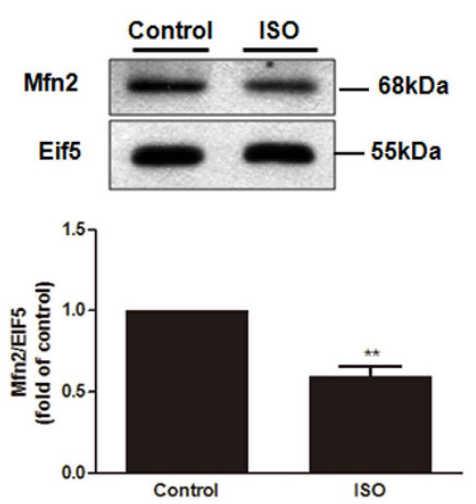

Figure 5. Mfn2 is the direct target gene of miR-214. (A) Conservation of the Mfn2 $3^{\prime}$ UTR binding site for miR-214 among different species and the mutated binding sites of Mfn2 3'UTR. (B) miR-214 targets the wild type but not the mutant $3^{\prime} \mathrm{UTR}$ of Mfn2. HEK293A cells were co-transfected with $\mathrm{pGL}_{4}-\mathrm{Mfn}^{2}-3^{\prime} \mathrm{UTR}$ and agomir-214/NC or the $\mathrm{pGL}_{4}-\mathrm{Mfn} 2-3^{\prime} \mathrm{UTR}$ mutant and agomir-214/NC for $48 \mathrm{~h}$; then, luciferase activity was detected $\left(\mathrm{n}=3\right.$, mean $\pm \mathrm{SEM},{ }^{* * *} \mathrm{P}<0.001$ vs. Mfn2 $3^{\prime} \mathrm{UTR}+$ agomir-NC, ${ }^{\# \# \#} \mathrm{P}<0.001$ vs. Mfn2 $3^{\prime} \mathrm{UTR}$ mutant + agomir-214). (C) MiR-214 did not affect Mfn2 mRNA expression, as determined by quantitative real-time PCR. (D) miR-214 negatively regulated the Mfn2 protein level in CFs, as determined by western blot analysis in CFs $\left(\mathrm{n}=4\right.$, mean $\pm \mathrm{SEM},{ }^{*} \mathrm{P}<0.05,{ }^{* * *} \mathrm{P}<0.001 v s$. NC). (E,F) Levels of Mfn 2 protein were downregulated in ISO-induced fibrotic heart tissue and CFs $\left(\mathrm{n}=4-6\right.$, mean $\pm \mathrm{SEM},{ }^{*} \mathrm{P}<0.05,{ }^{* * *} \mathrm{P}<0.001$ vs. Control). 
A
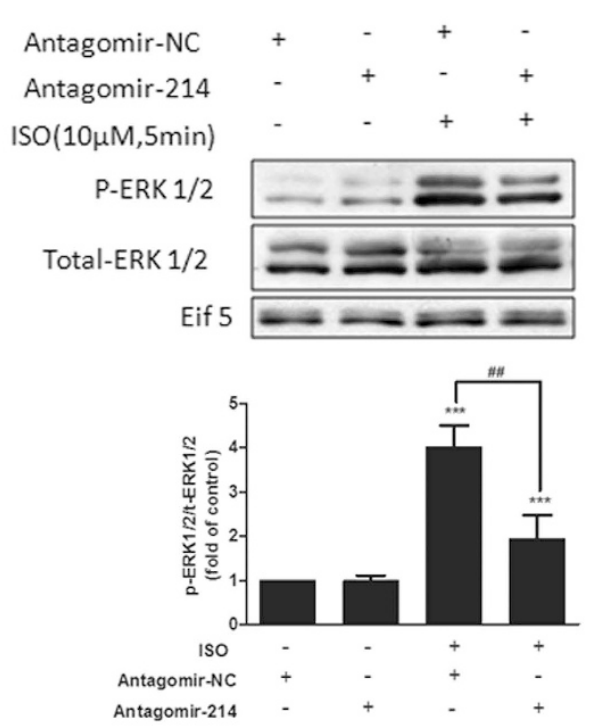

B
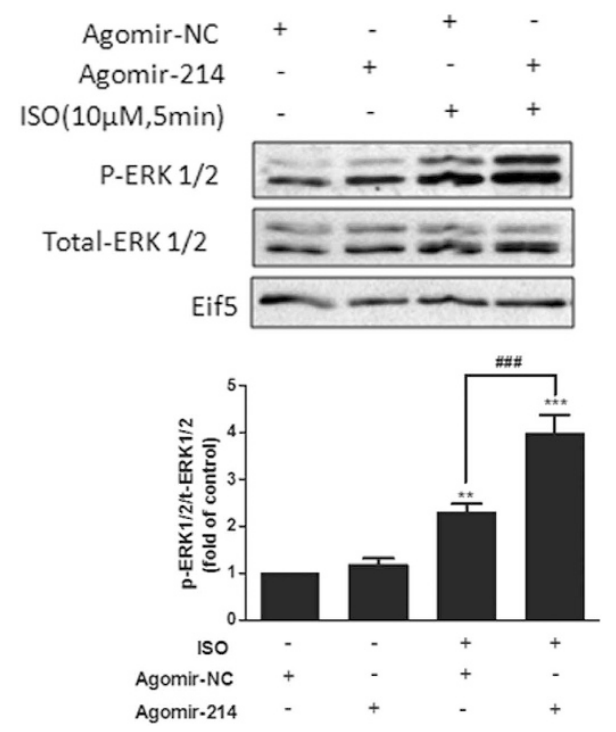

Figure 6. miR-214 positively regulates ISO-induced extracellular signal-regulated kinase 1/2 (ERK1/2) phosphorylation in CFs. (A) Downregulation of miR-214 inhibits ISO-induced ERK1/2 phosphorylation. CFs were transfected with antagomir-214, starved for $24 \mathrm{~h}$, and then incubated with ISO $(10 \mu \mathrm{M})$ for $5 \mathrm{~min}$. (B) Overexpression of miR-214 promoted ISO-induced ERK1/2 phosphorylation. CFs were transfected with agomir-214, starved for $24 \mathrm{~h}$, and then incubated with ISO $(10 \mu \mathrm{M})$ for $5 \mathrm{~min}$. The phosphorylation of ERK1/2 was analysed using western blotting $\left(\mathrm{n}=4\right.$, mean $\pm \mathrm{SEM},{ }^{*} \mathrm{P}<0.05,{ }^{* *} \mathrm{P}<0.001,{ }^{* * *} \mathrm{P}<0.001$ vs. Control).

\begin{tabular}{|c|c|c|}
\hline & Sense primers $\left(5^{\prime}-3^{\prime}\right)$ & Antisense primers $\left(5^{\prime}-3^{\prime}\right)$ \\
\hline $5 s$ & GTCTACGGCCATACCACCCTGAAC & \multirow{2}{*}{$\begin{array}{l}\text { Provided by miScript SYBR Green PCR Ki } \\
\text { (Tiangen Biotech, Beijing, China) }\end{array}$} \\
\hline miR-214 & ACAGCAGGCACAGACAGGCAG & \\
\hline Mfn2 & ATGATAGACGGCTTGAA & CGACTCCCTCTTTGTGA \\
\hline 18s rRNA & TACCACATCCAAGGAAGGCAGCA & TGGAATTACCGCGGCTGCTGGCA \\
\hline Collagen I & ATCAGCCCAAACCCCAAGGAGA & CGCAGGAAGGTCAGCTGGATAG \\
\hline Collagen III & TGATGGGATCCAATGAGGGAGA & GAGTCTCATGGCCTTGCGTGTTT \\
\hline
\end{tabular}

Table 1. List of primers used in qRT-PCR.

Real-Time PCR. Small RNAs were isolated using the miRcute miRNA isolation kit (Tiangen Biotech, Beijing, China). The miRcute microRNA first-strand cDNA synthesis and qPCR detection kits (Tiangen) were used for miRNA analysis. Quantitative PCR was performed using the ABI PRISM 7700 Sequence Detection System (Applied Biosystems, Invitrogen). Rat 5S ribosomal RNA was used as an internal control.

Total RNA was extracted using the TRIzol Reagent method (Invitrogen, Carlsbad, CA, USA). Relative quantification by real-time PCR was performed using SYBR Green-based detection of PCR products in real time with the ABI PRISM 7700 Sequence Detection System (Applied Biosystems, Invitrogen). Rat 18S ribosomal RNA was amplified as a reference standard. The reactions were prepared in triplicate and heated to $95^{\circ} \mathrm{C}$ for $5 \mathrm{~min}$, followed by 40 cycles of $94^{\circ} \mathrm{C}$ for $30 \mathrm{~s}, 60^{\circ} \mathrm{C}$ for $30 \mathrm{~s}$, and $72^{\circ} \mathrm{C}$ for $30 \mathrm{~s}$. The primer sequences are shown in Table 1 .

Western Blot. Total protein was extracted in RIPA buffer (CxBio, Shanghai, China) supplemented with phenylmethanesulfonyl (PMSF, CxBio). The protein concentrations were measured using the BCA Protein Assay (Applygen, Beijing, China). The samples were separated on a 10\% SDS-polyacrylamide gel and then transferred to a nitrocellulose membrane, which was blocked in 5\% skim milk for $1 \mathrm{~h}$ and then incubated with primary antibodies at $4{ }^{\circ} \mathrm{C}$ overnight. After the washing steps, the membranes were incubated with horseradish peroxidase-labelled secondary antibodies for $1 \mathrm{~h}$ at room temperature. The bands were visualized using a chemiluminescence detection system, and the densitometric results were analysed with Image J software. EIF5 levels were used as internal controls for protein normalization.

Anti-Mfn2 and anti-EIF5 for western blotting were purchased from Santa Cruz Biotechnology (CA, USA), and anti-phospho-ERK1/2 and anti-total-ERK1/2 antibodies were purchased from Cell Signalling Technology (Beverly, MA, USA).

Dual-Luciferase Reporter Assay. The sequence of the miR-214 predicted target region was synthesized from the 3'UTR of rat Mfn2 and cloned into the dual luciferase reporter vector pmiRGLO. A control construct with mutations incorporated in the miR-214 seed region was generated in a similar manner. The sequences of these constructs 
were verified. HEK293 cells were transfected with $200 \mathrm{ng}$ of plasmid DNA (wild-type PGL4-Mfn2-3'UTR/mutant PGL4-Mfn2-3'UTR which were commercially constructed using GeneChem (Shanghai GeneChem Co., Ltd.)) and agomir ( $50 \mathrm{nmol} / \mathrm{L}$; RibioBio) using Lipofectamine 2000 according to the manufacturer's instructions (Invitrogen). The luciferase activity was measured by the Dual-Luciferase Reporter Assay System (Promega, Madison, WI, USA).

${ }^{3} \mathrm{H}$-Proline Incorporation Assay. The collagen synthesis was determined by the quantification of ${ }^{3} \mathrm{H}$-Proline incorporation. In brief, CFs were plated on 24 -well culture dishes at a density of $5 \times 10^{4}$ cells/well and treated with or without various reagents, as mentioned above. The cells were labelled with ${ }^{3} \mathrm{H}$-Proline $(1 \mu \mathrm{Ci})$ for the last $24 \mathrm{~h}$, fixed in $10 \%$ trichloroacetic acid, and collected in $0.01 \mathrm{M} \mathrm{NaOH}$ and $1 \mathrm{~g} / \mathrm{L} \mathrm{SDS}$. Aliquots for each treatment were counted in a liquid scintillation counter using $3 \mathrm{ml}$ of scintillation fluid.

BrdU Incorporation Assay. Proliferation was assessed by the quantification of 5-bromo-2' -deoxyuridine (BrdU) incorporation. In brief, CFs were plated on 96-well culture dishes at a density of $1 \times 10^{4}$ cells/well and treated with or without various reagents, as mentioned above. Then, $\mathrm{BrdU}(10 \mu \mathrm{mol} / \mathrm{L})$ was added to the medium, and the cells were incubated for another $6 \mathrm{~h}$. Subsequently, the cells were fixed, and BrdU incorporation was determined with a Cell Proliferation ELISA Kit (Roche Diagnostics, Mannheim, Germany) according to the manufacturer's instructions.

Statistical Analysis. All of the experiments were performed at least three times. The data are expressed as means \pm SEMs and were analysed by ANOVA and a post-hoc Tukey's analysis or by a t-test as appropriate. A p value of 0.05 or less was considered significant.

\section{References}

1. Kong, P., Christia, P. \& Frangogiannis, N. G. The pathogenesis of cardiac fibrosis. Cell Mol Life Sci 71, 549-574 (2014)

2. Tao, H. et al. Epigenetic regulation of cardiac fibrosis. Cell Signal 25, 1932-1938 (2013).

3. Florea, V. G. \& Cohn, J. N. The Autonomic Nervous System and Heart Failure. Circ Res 114, 1815-1826 (2014).

4. Liu, N. et al. HIP-55/DBNL-dependent regulation of adrenergic receptor mediates the ERK1/2 proliferative pathway. Mol Biosyst 10, 1932-1939 (2014).

5. Lu, H. et al. Danshensu inhibits beta-adrenergic receptors-mediated cardiac fibrosis by ROS/p38 MAPK axis. Biol Pharm Bull 37, 961-967 (2014).

6. Chen, C. et al. beta-Adrenergic receptors stimulate interleukin-6 production through Epac-dependent activation of PKCdelta/p38 MAPK signalling in neonatal mouse cardiac fibroblasts. Br J Pharmacol 166, 676-688 (2012).

7. Kim, J., Eckhart, A. D., Eguchi, S. \& Koch, W. J. Beta-adrenergic receptor-mediated DNA synthesis in cardiac fibroblasts is dependent on transactivation of the epidermal growth factor receptor and subsequent activation of extracellular signal-regulated kinases. $J$ Biol Chem 277, 32116-32123 (2002).

8. Bauersachs, J. Regulation of myocardial fibrosis by MicroRNAs. J Cardiovasc Pharmacol 56, 454-459 (2010).

9. Da, C. M. P. et al. Conditional dicer gene deletion in the postnatal myocardium provokes spontaneous cardiac remodeling. Circulation 118, 1567-1576 (2008).

10. Chen, S. et al. Cardiac miR-133a overexpression prevents early cardiac fibrosis in diabetes. J Cell Mol Med 18, 415-421 (2014)

11. Castoldi, G. et al. MiR-133a regulates collagen 1A1: potential role of miR-133a in myocardial fibrosis in angiotensin II-dependent hypertension. J Cell Physiol 227, 850-856 (2012).

12. Limana, F. et al. HMGB1 attenuates cardiac remodelling in the failing heart via enhanced cardiac regeneration and miR-206-mediated inhibition of TIMP-3. PLoS One 6, e19845 (2011).

13. Roy, S. et al. MicroRNA expression in response to murine myocardial infarction: miR-21 regulates fibroblast metalloprotease- 2 via phosphatase and tensin homologue. Cardiovasc Res 82, 21-29 (2009).

14. Thum, T. et al. MicroRNA-21 contributes to myocardial disease by stimulating MAP kinase signalling in fibroblasts. Nature 456, 980-984 (2008).

15. Zhang, Y. et al. miR-29b as a therapeutic agent for angiotensin II-induced cardiac fibrosis by targeting TGF-beta/Smad3 signaling. Mol Ther 22, 974-985 (2014).

16. Zhu, J. N. et al. Smad3 inactivation and MiR-29b upregulation mediate the effect of carvedilol on attenuating the acute myocardium infarction-induced myocardial fibrosis in rat. PLoS One 8, e75557 (2013).

17. Hou, Y. et al. beta-adrenoceptor regulates miRNA expression in rat heart. Med Sci Monit 18, R309-R314 (2012).

18. van Rooij, E. et al. A signature pattern of stress-responsive microRNAs that can evoke cardiac hypertrophy and heart failure. Proc Natl Acad Sci USA 103, 18255-18260 (2006).

19. Denby, L. et al. MicroRNA-214 Antagonism Protects against Renal Fibrosis. J Am Soc Nephrol 25, 65-80 (2014).

20. Chen, K. H. et al. Role of mitofusin 2 (Mfn2) in controlling cellular proliferation. Faseb J 28, 382-394 (2014).

21. Ikeda, S. et al. Altered microRNA expression in human heart disease. Physiol Genomics 31, 367-373 (2007).

22. Duan, Q. L. et al. MicroRNA-214 Is Upregulated in Heart Failure Patients and Suppresses XBP1-Mediated Endothelial Cells Angiogenesis. J Cell Physiol 230, 1964-1973 (2015).

23. Yang, T. et al. MicroRNA-214 provokes cardiac hypertrophy via repression of EZH2. Biochem Biophys Res Commun 436, 578-584 (2013).

24. Aurora, A. B. et al. MicroRNA-214 protects the mouse heart from ischemic injury by controlling $\mathrm{Ca}(2)(+)$ overload and cell death. J Clin Invest 122, 1222-1232 (2012).

25. Lv, G. et al. MicroRNA-214 protects cardiac myocytes against H2O2-induced injury. J Cell Biochem 115, 93-101 (2014).

26. Yang, H. et al. MicroRNA expression profiling in human ovarian cancer: miR-214 induces cell survival and cisplatin resistance by targeting PTEN. Cancer Res 68, 425-433 (2008).

27. Zhang, X. J. et al. Dysregulation of miR-15a and miR-214 in human pancreatic cancer. J Hematol Oncol 3, 46 (2010).

28. Yang, Z. et al. MicroRNA-214 is aberrantly expressed in cervical cancers and inhibits the growth of HeLa cells. Iubmb Life 61, 1075-1082 (2009).

29. Wang, X. et al. MiR-214 inhibits cell growth in hepatocellular carcinoma through suppression of beta-catenin. Biochem Biophys Res Commun 428, 525-531 (2012).

30. Misiewicz-Krzeminska, I. et al. Restoration of microRNA-214 expression reduces growth of myeloma cells through positive regulation of P53 and inhibition of DNA replication. Haematologica 98, 640-648 (2013).

31. Yang, T. et al. Cardiac hypertrophy and dysfunction induced by overexpression of miR-214 in vivo. J Surg Res 192, 317-325 (2014).

32. Coll, M. et al. Integrative miRNA and Gene Expression Profiling Analysis of Human Quiescent Hepatic Stellate Cells. Sci Rep. 5, 11549 (2015).

33. Zhang, M., Wang, X., Li, W. \& Cui, Y. miR-107 and miR-25 simultaneously target LATS2 and regulate proliferation and invasion of gastric adenocarcinoma (GAC) cells. Biochem Biophys Res Commun. 460, 806-812 (2015). 
34. Chen, K. C. et al. OxLDL up-regulates microRNA-29b, leading to epigenetic modifications of MMP-2/MMP-9 genes: a novel mechanism for cardiovascular diseases. FASEB J. 25, 1718-1728 (2011).

35. Ishteiwy, R. A., Ward, T. M., Dykxhoorn, D. M. \& Burnstein, K. L. The microRNA-23b/-27b cluster suppresses the metastatic phenotype of castration-resistant prostate cancer cells. PLoS One. 7, e52106 (2012).

36. Guo, R., Gu, J., Zhang, Z., Wang, Y. \& Gu, C. MicroRNA-410 functions as a tumor suppressor by targeting angiotensin II type 1 receptor in pancreatic cancer. IUBMB Life. 67, 42-53 (2015).

37. Yu, S. et al. miR-326 Targets Antiapoptotic Bcl-xL and Mediates Apoptosis in Human Platelets. PLoS One. 10, e0122784 (2015).

38. de Brito, O. M. \& Scorrano, L. Mitofusin-2 regulates mitochondrial and endoplasmic reticulum morphology and tethering: the role of Ras. Mitochondrion 9, 222-226 (2009).

39. Tang, W. X., Wu, W. H., Zeng, X. X., Bo, H. \& Huang, S. M. Early protective effect of mitofusion 2 overexpression in STZ-induced diabetic rat kidney. Endocrine 41, 236-247 (2012)

40. Pages, G. et al. Mitogen-activated protein kinases p42mapk and p44mapk are required for fibroblast proliferation. Proc Natl Acad Sci USA 90, 8319-8323 (1993).

41. Hayashida, T., Decaestecker, M. \& Schnaper, H. W. Cross-talk between ERK MAP kinase and Smad signaling pathways enhances TGF-beta-dependent responses in human mesangial cells. Faseb J 17, 1576-1578 (2003).

42. Simpson, P. \& Savion, S. Differentiation of rat myocytes in single cell cultures with and without proliferating nonmyocardial cells. Cross-striations, ultrastructure, and chronotropic response to isoproterenol. Circ Res 50, 101-116 (1982).

\section{Acknowledgements}

This work was supported by the National Basic Research Program of China (No. 2014CBA02000) and the National Natural Science Foundation of China (No. 81270157, 81370317, 81471893, 91539123 and 81100164), the Research Fund for the Doctoral Program of Higher Education (No. 20110001120015).

\section{Author Contributions}

M.S. and H.Y.Y. researched data and participated in writing of the manuscript. Y.Y.Z. and Z.J.L. contributed to the discussion. H.Y.Y. and Z.J.L. provided oversight for the project and participated in editing of the manuscript. W.G. is the guarantor of this work.

\section{Additional Information}

Competing financial interests: The authors declare no competing financial interests.

How to cite this article: Sun, M. et al. MicroRNA-214 Mediates Isoproterenol-induced Proliferation and Collagen Synthesis in Cardiac Fibroblasts. Sci. Rep. 5, 18351; doi: 10.1038/srep18351 (2015).

(c) (i) This work is licensed under a Creative Commons Attribution 4.0 International License. The images or other third party material in this article are included in the article's Creative Commons license, unless indicated otherwise in the credit line; if the material is not included under the Creative Commons license, users will need to obtain permission from the license holder to reproduce the material. To view a copy of this license, visit http://creativecommons.org/licenses/by/4.0/ 